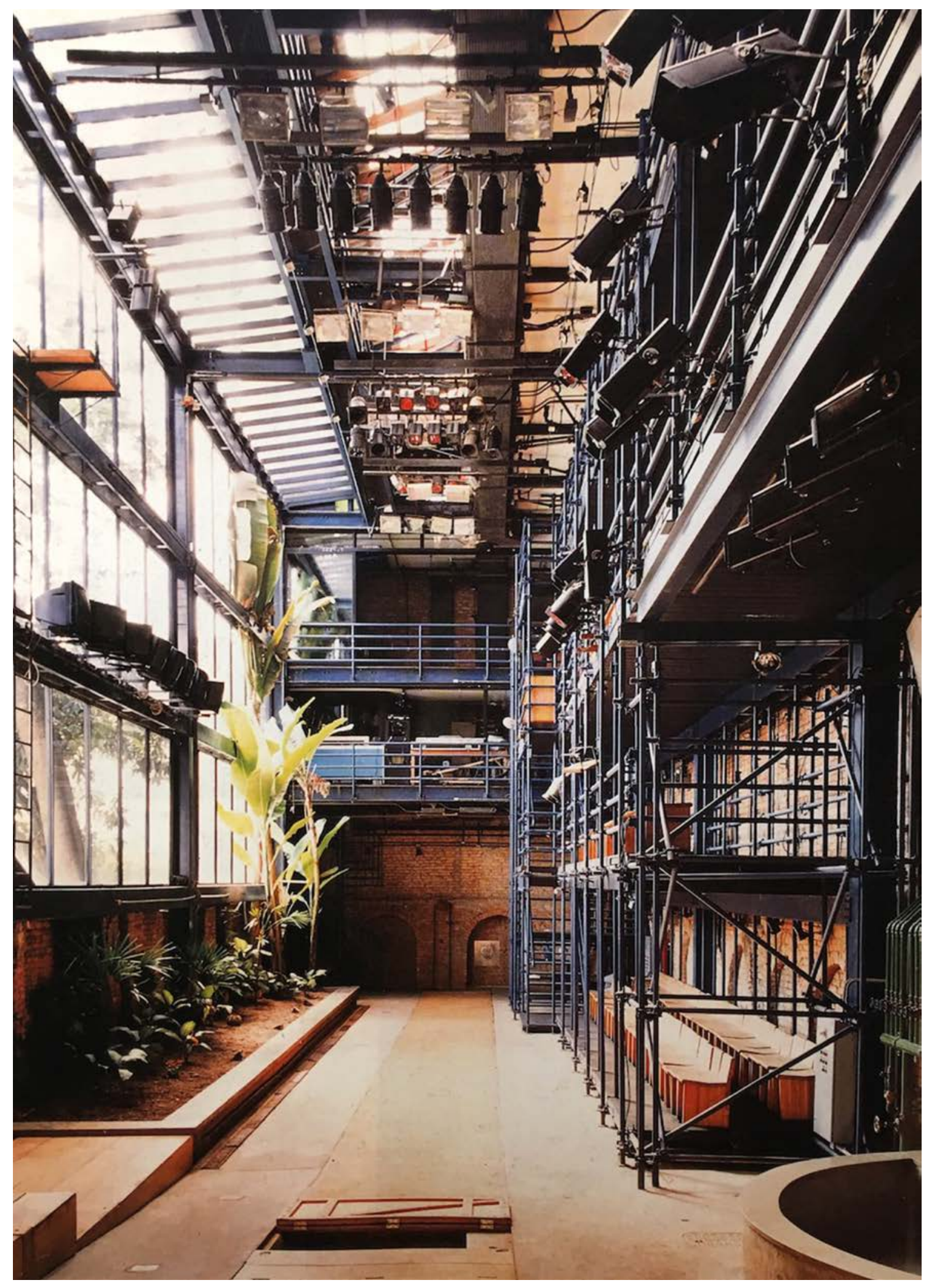

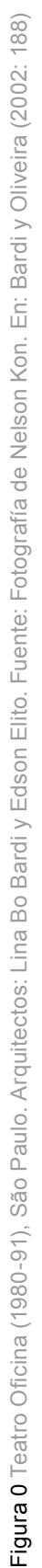



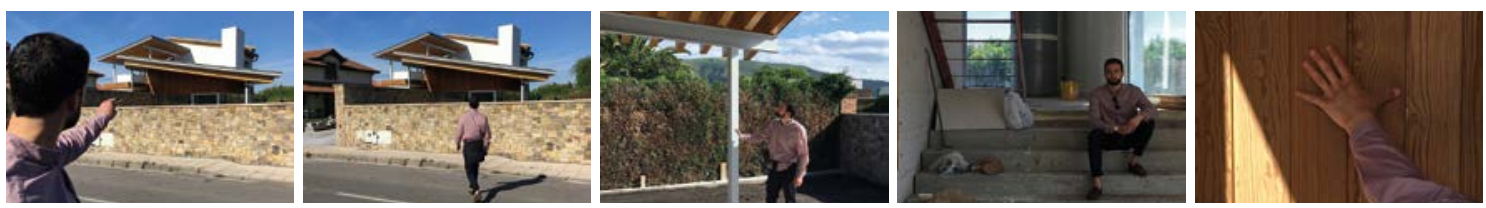

\title{
INTERIORES EXTERIORIZADOS, TRES CASOS DE ESTUDIO EN LA ARQUITECTURA BRASILEÑA: MENDES DA ROCHA, LINA BO BARDI E ISAY WEINFELD'
}

\author{
EXTERIORIZED INTERIORS, THREE CASE STUDIES IN BRAZILIAN ARCHITECTURE: \\ MENDES DA ROCHA, LINA BO BARDI AND ISAY WEINFELD'
}

Héctor Navarro Martinez², Manuel Blanco Lage ${ }^{3}$

RESUMEN

\begin{abstract}
El presente artículo expone tres estudios de caso, en los que espacios interiores de diferentes usos han sido proyectados con la intención de generar una concepción espacial basada en lo urbano. Paulo Mendes da Rocha, Lina Bo Bardi e Isay Weinfeld consiguen, con sus propuestas, exteriorizar espacios interiores. Detrás de los distintos recursos arquitectónicos utilizados, existe un discurso que busca impregnar el recinto interior de referencias con temas vinculados a la dimensión urbana. Para realizar con éxito tales operaciones, es necesario modificar el concepto de uso y el entendimiento de lo que puede ser una vivienda, una tienda o un teatro. Con ello, se consigue deshacer los límites canónicos entre espacio público y arquitectura, creando una relación simbiótica que beneficia a ambas partes y al concepto de ciudad como espacio de encuentro. La arquitectura se convierte en la herramienta capaz de propiciar la vida comunitaria, desdibujando los límites entre lo privado y lo público, y desarticulando la privatización del espacio.
\end{abstract}

Palabras clave: Mendes da Rocha, Lina Bo Bardi, Isay Weinfeld, arquitectura brasileña, espacio público.

\section{ABSTRACT}

This article presents three case studies in which interior spaces with different uses have been planned with the intention of generating an urban spatial conception. Paulo Mendes da Rocha, Lina Bo Bardi and Isay Weinfeld achieve the exteriorization of interior spaces in their designs. Behind the different architectural resources used, there is a discourse that seeks to impregnate the interior space with references to urban-linked themes. To successfully carry out such operations, it is necessary to modify the concept of use and understanding of what can be a dwelling, a store or a theater. This is achieved by undoing the canonical boundaries between public space and architecture, creating a symbiotic relationship that benefits both parties and the concept of cities as meeting spaces. Architecture becomes the tool capable of fostering community life, blurring the boundaries between that which is private and public, and disarticulating the privatization of space.

Keywords: Mendes da Rocha, Lina Bo Bardi, Isay Weinfeld, Brazilian architecture, public space.

Artículo recibido el 23 de febrero de 2017 y aceptado el 6 de junio de 2017 DOI: https://doi.org/10.22320/07196466.2017.35.051.02

[1] Este artículo está basado en los resultados de investigación de la tésis doctoral en desarrollo "Simbiósis y fronteras entre Arquitectura y espacio urbano contemporáneo", realizada en la Escuela Técnica Superior de Arquitectura, Universidad Politécnica de Madrid, España, del autor Héctor Navarro Martinez.

[2] Escuela Técnica Superior de Arquitectura, Universidad Politécnica de Madrid, España. hectornavarro@me.com

[3] Escuela Técnica Superior de Arquitectura, Universidad Politécnica de Madrid, España. manuel.blanco@upm.es 
A priori, el espacio público está supeditado a estrategias de calado político que implican intervenciones urbanísticas capaces de mejorar y dotar a la ciudad de espacios de calidad. Sin embargo, el presente texto pone en valor ciertas actuaciones que operan desde el interior de la pieza arquitectónica, las cuales tienen una repercusión equiparable a la de cualquier estrategia urbana. Es decir, la figura del arquitecto, que tiene restringida su labor a los espacios arquitectónicos, también puede ofrecer una positiva labor con repercusiones directas en la calidad urbana y el bien comunitario. Para desarrollar este tema, se analizan tres casos destacados cuya capacidad de hacer más público un espacio que se presupone privado, da buena muestra de cómo estos planteamientos no están supeditados tampoco a usos concretos. Así, Lina Bo Bardi actúa en un teatro que quiere volver a conectar con el pueblo y recuperar la esencia de los terreiros ${ }^{4}$ históricos de Salvador de Bahía. Isay Weinfeld plantea recuperar la esencia de la marca de sandalias havaianas, cuyos puntos de venta más primitivos eran los mercados, donde, por lo tanto, la calle formaba parte de la experiencia de compra. Y el caso de Paulo Mendes da Rocha ilustra muy bien cómo incluir una dimensión urbana incluso en un espacio privado destinado a vivienda.

Muchas propuestas como las que aquí se exponen constituyen "caminos de vuelta" que pretenden recuperar experiencias que pertenecían a la ciudad. En ese sentido, es imprescindible entender el funcionamiento del modelo socio-económico occidental, basado en la privatización y el individualismo, que poco a poco ha ido desvinculando de la dimensión pública a muchas actividades que originalmente se vinculaban a la ciudad. Se pueden identificar multitud de casos a lo largo de la historia, por ejemplo, la fuente pública o el lavadero popular, antes de que se incorporasen instalaciones de agua en la vivienda. A medida que la ciudad fue evolucionando y tecnificándose, muchas actividades se integraron al ámbito privado en aras del bienestar y la comodidad del ser humano. Pero además, con la privatización y la explotación económica de todos los estamentos públicos, otras actividades se profesionalizaron y se convirtieron en nuevas fuentes de negocio, como ocurrió con el deporte, el teatro o la música. Con el paso del tiempo, todas aquellas actividades prestadas del ámbito urbano sedimentan y enmascaran su origen primario, y con ello, también su significado, siendo el sector ciudadano con menos recursos económicos el más afectado en este proceso. Con el incremento del valor asociado a aquellas actividades, aparecen nuevos códigos y símbolos en la definición espacial que, en muchos casos, marginan y excluyen. 
Sin embargo, algunos críticos como Yoshio Futagawa apuntan a que en algunas ciudades se empieza a dar una evolución inversa. Las grandes urbes, con el precio del metro cuadrado de suelo cada vez más alto, deben prescindir de todos aquellos servicios que puede asumir la ciudad. Más aún, si se tiene en cuenta la tendencia a los modelos $24 / 7^{5}$. En ciudades consolidadas de densidad media-alta con dichas condiciones, cada vez es más habitual convertir el supermercado abierto 24 horas en una extensión de la propia casa o enviar la ropa a lavanderías cercanas. De ese modo, se puede prescindir del espacio que ciertas actividades ocupan dentro de la casa $y$, por consiguiente, se potencia la simbiosis $^{6}$ entre arquitectura y ciudad (Futagawa, 2007). Esta tendencia también ha sido reconocida de una manera más primitiva por Watsuji, quien ve en el modelo occidental de ciudad europea un modo de habitar en el que no existe casa, sino individuo y sociedad. A ojos del antropólogo, la configuración urbana de la ciudad permitía hacer uso de ésta como una extensión de la propia vivienda. Entonces, ve la cafetería como un cuarto de estar y la calle como un pasillo de la vivienda. A otra escala, Watsuji entiende la ciudad occidental como una gran casa (2006: 199).

\section{MÉTODOS}

Durante las primeras décadas del siglo $\mathrm{XX}$, Brasil desarrolló un conjunto arquitectónico donde el discurso general planteaba la búsqueda de un lenguaje arquitectónico propio que sintonizase con el pueblo brasileño y el Movimiento Moderno. El "Brazilian Style", representado en figuras como Lucio Costa o Affonso Eduardo Reidy, forma parte de esa primera fase de desarrollo. La nueva arquitectura se sentía propia dentro de un contexto político de cambio liderado por Getúlio Vargas (Andreoli y Forty, 2004). Generaciones posteriores pertenecientes a la Escuela Paulista, además de definir una obra reconocible cuyas señas de identidad eran la estructura, la técnica y el hormigón, buscaron en la arquitectura un aliado de la sociedad civil. Mendes da Rocha o Lina Bo Bardi dieron un sentido social al papel del arquitecto. Buscaban con sus propuestas educar, transmitir valores y recuperar lo comunitario, perdido con la privatización asociada a la sociedad colonial y la posterior industrialización (Montezuma, 2002). Y es ahí donde radica el interés de esta investigación que, llevando a cabo un estudio detallado de la obra de los arquitectos señalados, ha subrayado aquellos casos que pueden ser entendidos como "interiores exteriorizados". A través de los proyectos destacados, se analizan los mecanismos capaces de deshacer todos aquellos códigos relacionados con la privatización del espacio, que no hacen sino reforzar su condición como espacio interior, lo que, naturalmente, tiene una repercusión directa en la definición del espacio público. Las obras expuestas en el presente artículo consiguen, mediante su definición arquitectónica, borrar todo aquello que disocia interior y exterior. De esta manera, el espacio público no queda restringido a entornos propios del paisaje urbano, y se incorpora dentro de la arquitectura, la cual ha sido desvinculada del espacio público por la coyuntura histórica y sociocultural (Gutiérrez, 1983).

\section{RESULTADOS}

Teatro Oficina y la vivienda diseñada por Paulo Mendes da Rocha para su propia familia han sido los proyectos seleccionados como más representativos de la segunda mitad del siglo XX, en cuanto a los temas desarrollados aquí. Sin embargo, es importante entender que este tipo de iniciativas no tienen porqué estar vinculadas a un pensamiento más progresista o izquierdista, razón por la cual se ha ampliado la investigación hasta la actualidad para identificar e incorporar otro caso que ignore la causa social, y aquel acto de "exteriorizar" pueda enmarcarse en otro contexto. Es el caso de Isay Weinfeld, quien busca a través de las decisiones de proyecto la apertura del interior, sin ningún tipo de elemento que debilite la continuidad con el espacio público. No obstante, este caso tiene detrás un interés de negocio y las intenciones del arquitecto buscan que la filosofía de marca como producto popular sea coherente con la experiencia de compra. Independientemente del objetivo, la realidad es que esta acción puede ser tan beneficiosa para la ciudad como los otros dos proyectos destacados. De esta forma, todos ellos representan un abanico de contextos diferentes que han encontrado en un nuevo planteamiento, una propuesta espacial beneficiosa para la comunidad volviendo a sus orígenes. Y lo que es más importante, no confiando dicho cometido únicamente a intervenciones en la envolvente, sino también a acciones configuradas desde el interior. Se trata, por tanto, de espacios donde el vínculo con el texto urbano deriva de las decisiones más primordiales del proyecto $y$ atienden a conceptos espaciales estrechamente ligados a un uso concreto. 


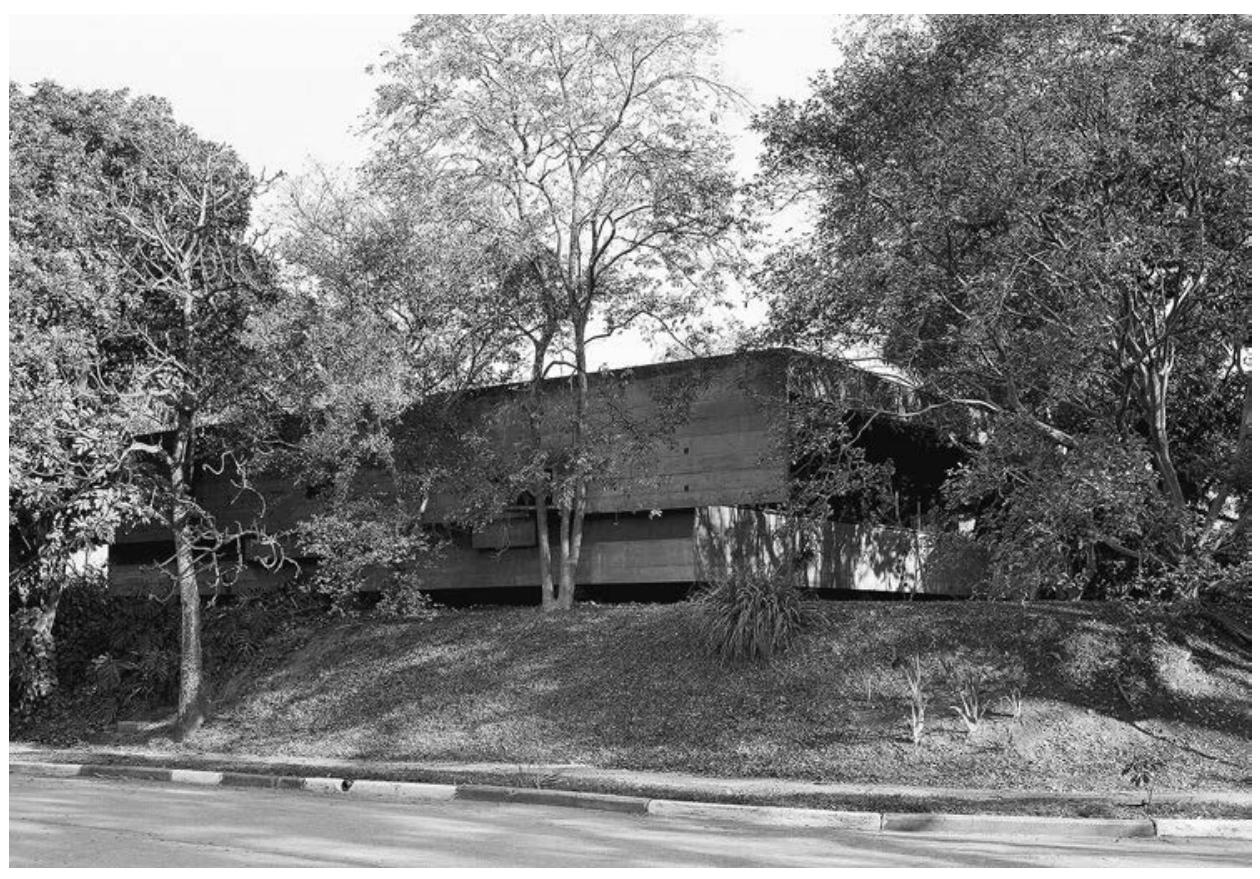

Figura 1 Casa del arquitecto (1964), São Paulo. Arquitecto: Paulo Mendes da Rocha. Fuente: Fotografía de Nelson Kon (http://www.nelsonkon.com.br)

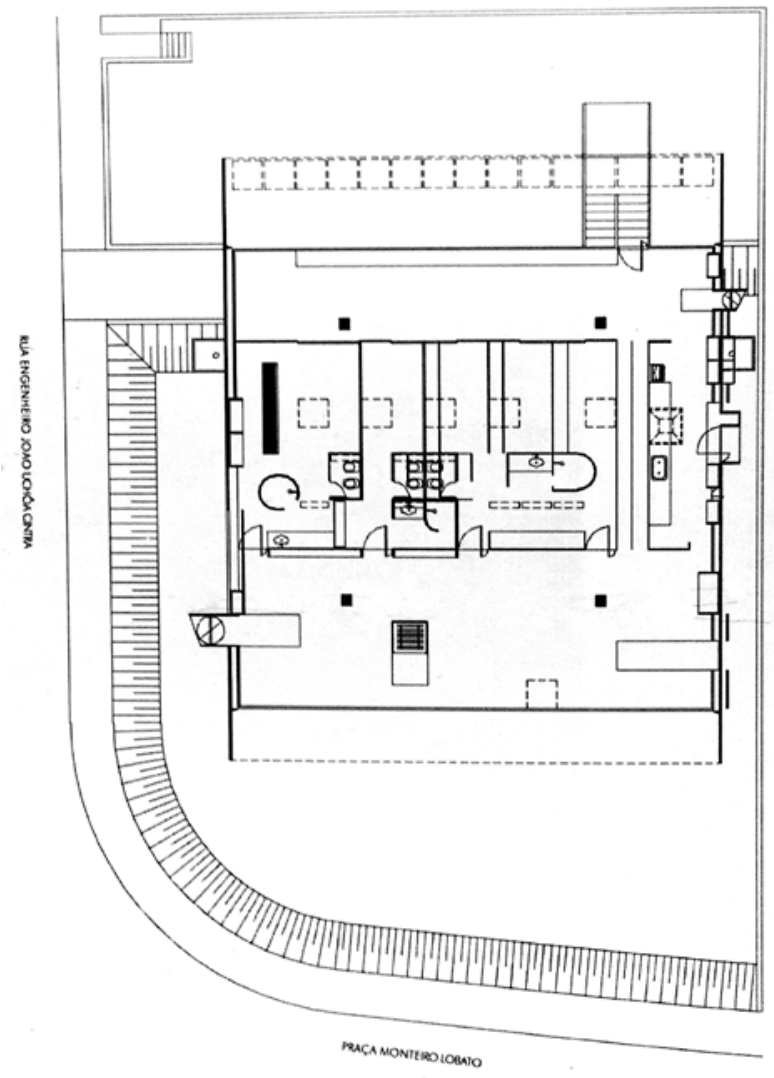

Figura 2 Casa del arquitecto (1964), São Paulo. Arquitecto: Paulo Mendes da Rocha. Fuente: Piñón (2003: 48) 
Paulo Mendes da Rocha y la inclusión de la ética social en el espacio residencial

La figura de João Vilanova Artigas fue imprescindible en la formación de una generación conocida como la 'Escuela Paulista', la cual surgió como respuesta a una situación política que tenía repercusiones directas en la libertad de la sociedad'. La Escuela de Arquitectura de São Paulo (FAU-USP), donde impartía clase junto a Paulo Mendes da Rocha, había nacido de la escuela de ingeniería y Vilanova Artigas se encargó de su diseño y construcción. La misma escuela se convirtió en un edificio-manifiesto desde el que se inculcó a las futuras generaciones de arquitectos una manera de acometer la arquitectura que siempre buscaba el bien común y la puesta en valor de los derechos civiles. Los círculos intelectuales criticaron activamente la intrusión de Estados Unidos tanto a nivel cultural como económico. Estas élites veían el 'Brazilian Style' como una expresión de sumisión ante el imperialismo, donde hubo un papel activo de promoción por parte de los norteamericanos. En diversas publicaciones de Vilanova Artigas se puede detectar esta opinión: "La Arquitectura Moderna de Brasil avanza de tal manera que sirve de propaganda a cualquier villanía comercial, [...] al tiempo que refuerza la penetración del imperialismo, dándole cobertura para entrar sin ser notado por las puertas de los movimientos culturales" (Deckker, 2001: 200).

A través de la obra Paulo Mendes da Rocha se pueden identificar los temas que desarrolló la 'Escuela Paulista', muchos de ellos, promovidos por Vilanova Artigas: la deformación topográfica de la cota cero; la volumetría unitaria cuya envolvente planteaba una postura impermeable que contrastaba con interiores de gran riqueza y complejidad espacial; un lenguaje estructural que definía forma y relación interior-exterior; y la técnica aplicada al detalle constructivo.

La arquitectura, desde esta perspectiva, debía incluir una labor social y el papel del arquitecto debía orientarse a velar por los intereses del pueblo oprimido. Dentro de las muchas aportaciones que se pueden distinguir en la obra de Mendes da Rocha, las siguientes líneas tienen como intención poner en valor las operaciones espaciales que desarrolló en su propia vivienda, proyectada entre 1963 y 1964. Proyectó dos casas gemelas: para la familia de su hermana y la suya propia (Figura 1). En ambos casos, se trata de un volumen de hormigón elevado, donde se libera el plano del suelo generando un espacio en sombra (Figura 3). El proyecto parece recoger las enseñanzas de Le Corbusier, quien abogaba por una arquitectura separada del suelo por pilotis, facilitando el flujo de lo natural. Sin embargo, una vivienda es una propiedad privada. El arquitecto planteó una solución en el perímetro de la parcela que incluye la manipulación topográfica del límite. Con ello, se evita elementos verticales que supongan interrupciones más evidentes y abruptas. Estas acciones reelaboran el límite canónico y plantean un modelo de ciudad donde el plano de suelo fluye y no se ve interrumpido (García del Monte, 2011:151-158).

Muchos de los arquitectos de la 'Escuela Paulista' buscaban en sus proyectos residenciales hacer una crítica acérrima a la vivienda burguesa y aportar soluciones para la lucha de clases. Intentaban deshacer la compartimentación tan rígida de los espacios, alejarse de la estructura espacial de los sobrado ${ }^{8}$ o incluso crear viviendas sin ala de servicio, algo que para arquitectos como Vilanova Artigas representaba una actualización de la esclavitud colonial. Se organizó la vivienda en tres bandas (Figura 2). En uno de los laterales se ubican las zonas comunes; un espacio amplio y generoso, bien iluminado y protegido por la cubierta en vuelo. En fachada, se incluye unos mecanismos que facilitan la ventilación, consiguiendo que diseño y técnica definan un estilo propio. En la banda paralela opuesta, se desarrolla un espacio libre, que funciona como pasillo de acceso a las unidades de dormitorios, pero también como zona de estudio. Las dimensiones, así como las condiciones de luz y ventilación, permiten esta duplicidad de uso (Figura 4).

Entre ambas piezas se encuentra la zona de dormitorios, sin contacto directo con la fachada, a excepción del dormitorio principal. Ahora bien, la operación a destacar en este artículo es el tratamiento de los límites de estos espacios. Tanto la tabiquería que separa los dormitorios, como los paramentos que independizan con respecto a las zonas comunes laterales no llegan al techo (Figura 5). Este detalle tiene muchas consecuencias en el funcionamiento de la vivienda. Mendes da Rocha imprime en ella algo que va más allá de lo espacial: plantea una vivienda para acoger un modelo de familia cuyas relaciones entre sus distintos integrantes lleva implícito un trasfondo social. La relación espacial entre las distintas unidades de dormitorios, donde se puede sentir la presencia del colindante, es similar a lo que ocurre en las favelas, cuya definición constructiva tiene una sensación mucho más comunitaria. De alguna manera, se está urbanizando la vida doméstica, reduciendo considerablemente la intimidad personal y desdibujando los límites clásicos

[7] La noche del 31 de marzo de 1964 tuvo lugar el Golpe de Estado contra el presidente João Goulart, realizado por militares brasileños con el apoyo del gobierno de los Estados Unidos. Más tarde, el 13 de diciembre de 1968, la dictadura militar brasileña que había tomado el poder cuatro años antes, proclamó el Acto Institucional №5, un decreto por el que se disolvía el Congreso Nacional se censuraban los medios de comunicación y se restringían enormemente los derechos civiles, asumiéndose la tortura como práctica represiva. Después de este hecho histórico y hasta el final del gobierno del general Médici, en 1974, fueron muchos los que perteneciendo a círculos intelectuales -artistas, estudiantes, arquitectos, etc.- fueron apresados, torturados, asesinados o forzados al exilio. Dos arquitectos que padecieron esta represión fueron Vilanova Artigas y Paulo Mendes da Rocha, quienes fueron desvinculados de la Universidad de São Paulo y a quienes también se les prohibió ejercer la profesión en el país.

[8] El sobrado era la vivienda por excelencia desde época colonial, con dos o más pisos en los que se organizaban las distintas partes de la residencia. Se construían en hileras paralelas a las calles que se adaptaban a la caprichosa geografía y, como consecuencia directa, se conformaban paisajes urbanos heterogéneos parecidos a las tramas urbanas de pueblos y centros históricos de ciudades portuguesas (Gutiérrez, 1983:72) 


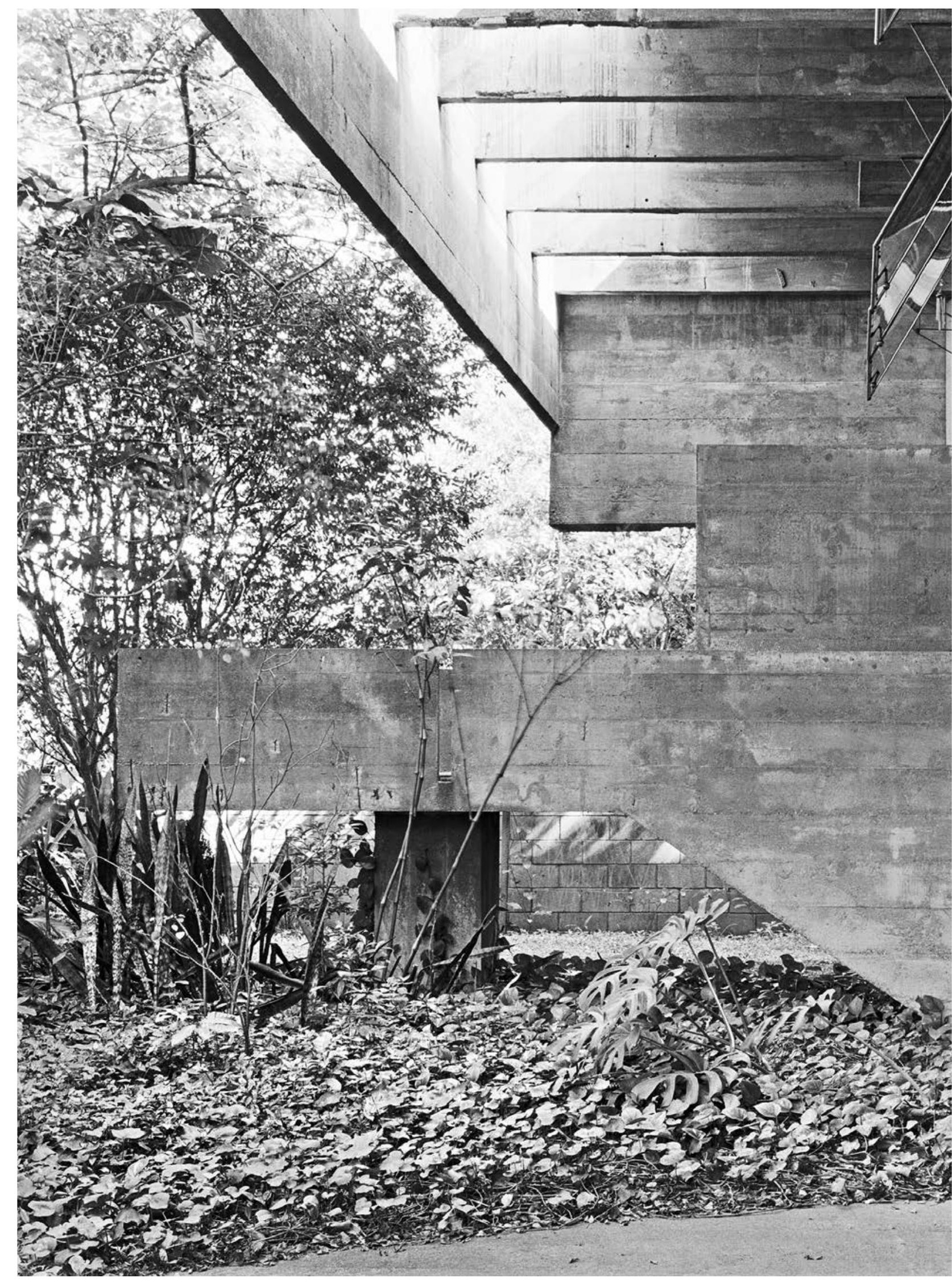

Figura 3 Casa del arquitecto (1964), São Paulo. Arquitecto: Paulo Mendes da Rocha. Fuente: Fotografía de Nelson Kon. En: Mendes da Rocha (2013 


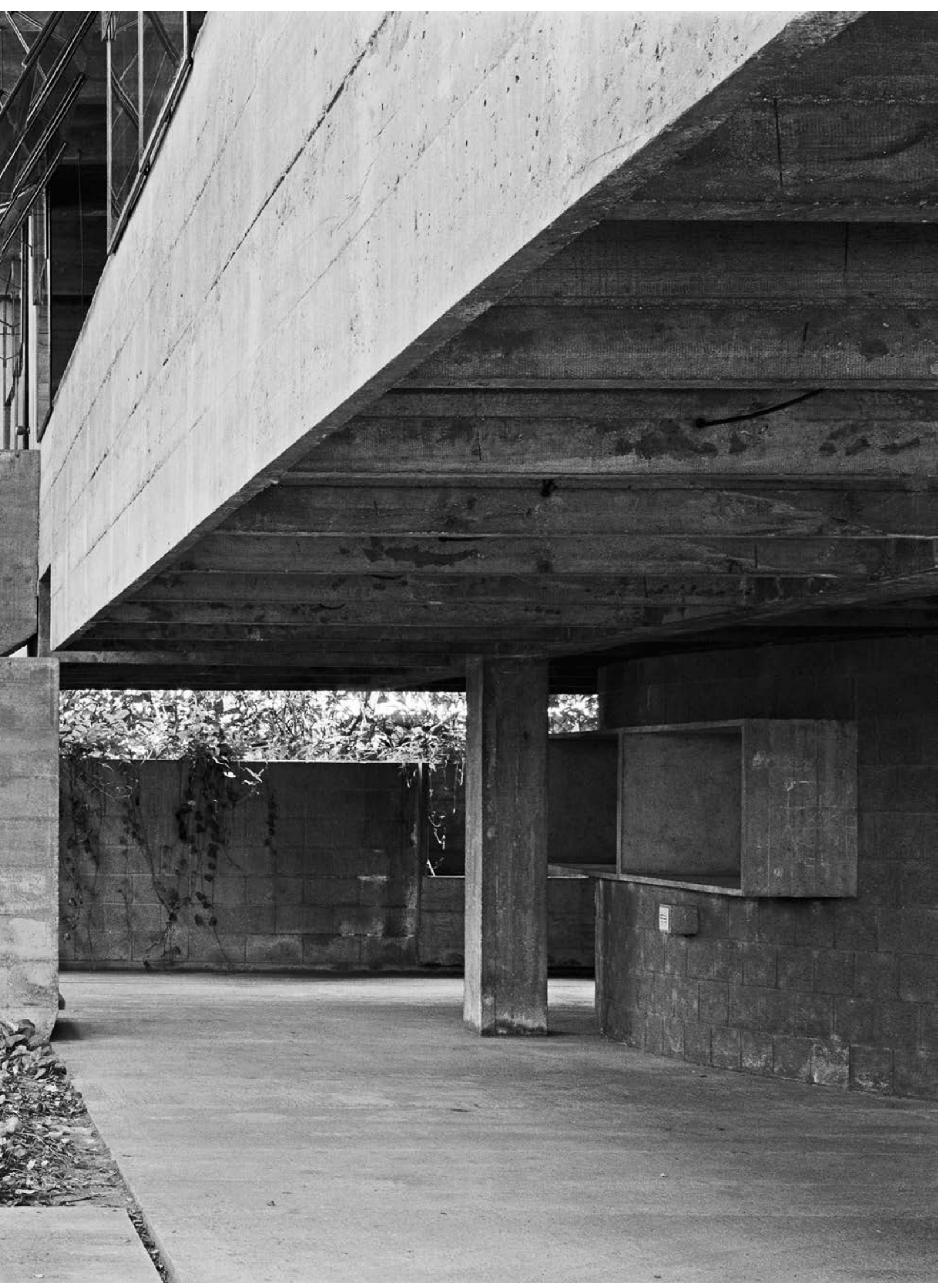

40) 

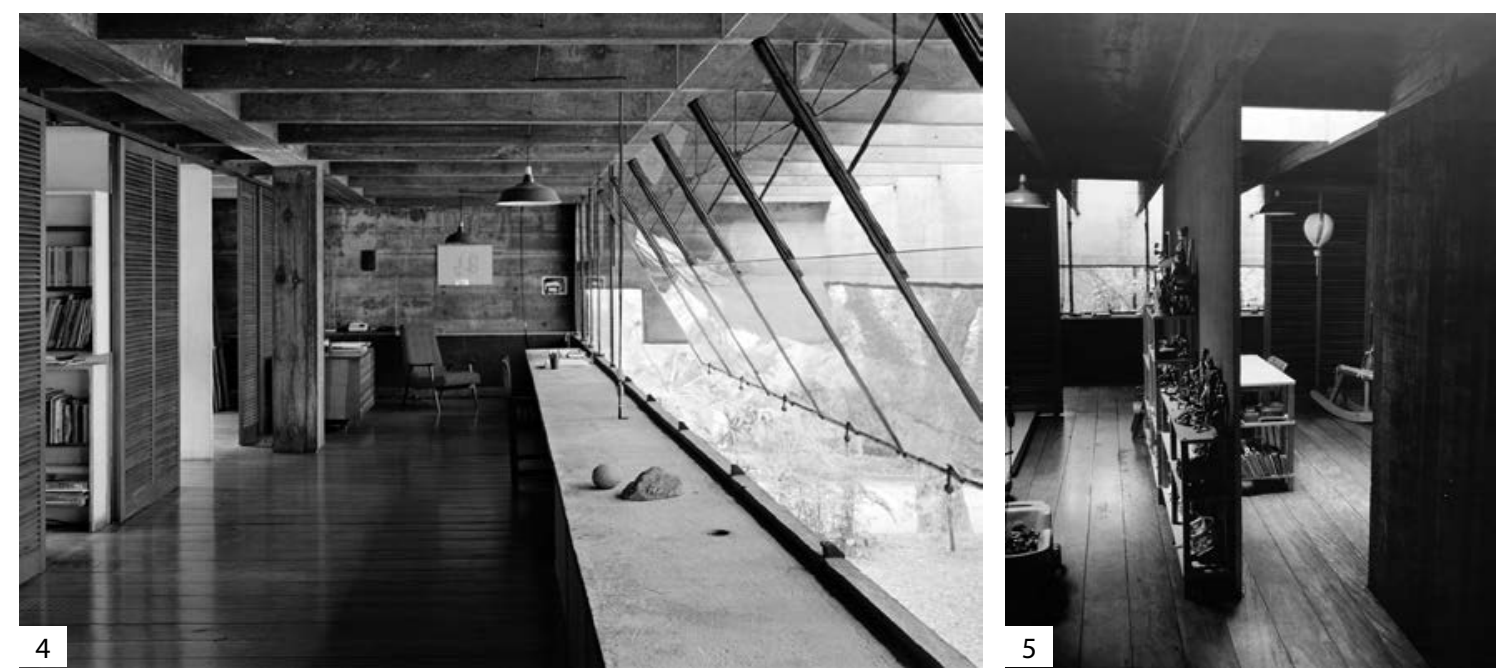

Figura 4 Casa del arquitecto (1964), São Paulo. Arquitecto: Paulo Mendes da Rocha. Fuente: Fotografía de Nelson Kon (http://www nelsonkon.com.br) / Figura 5 Casa del arquitecto (1964), São Paulo. Arquitecto: Paulo Mendes da Rocha. Fuente: Fotografía de Helio Piñón. En: Piñón, 2003: 60)

del concepto de hogar. Flávio Motta se refiere a esta vivienda como un trabajo que alude a la idea de «vivir en mitad de una favela racionalizada» (Masao, 2004:162). La rigidez espacial de la casa burguesa basada en el aislamiento individual, provisto por el dormitorio de límites abruptos, deriva hacia una perspectiva colectiva y pública. Y los mismos principios que rigen el buen funcionamiento de una sociedad, se trasladan a la vivienda: la libertad de cada uno depende de la libertad del otro. Las normas asociadas a la ética social imprimen un orden en los colectivos humanos y tienen como resultado casas totalmente exteriorizadas pero espacialmente introvertidas.

El arquitecto consiguió manipular los límites dentro de la vivienda para establecer nuevas relaciones dentro de la estructura familiar. A la vez que hace una crítica al individualismo que rige la familia burguesa, consigue fortalecer los lazos familiares. Con una simple acción, trae a colación todo un debate sobre la familia. No importa si es una crítica hacia la burguesía o una referencia a una construcción tradicional como las favelas. Lo importante es que impregna el recinto espacial doméstico de valores que tienen que ver con la ética social, con aquello que hace que una comunidad se desarrolle mediante un correcto funcionamiento y una sensibilización de cada uno de los seres como entes individuales 9 .

\section{Lina Bo Bardi, vuelta al teatro popular}

La obra de Lina Bo Bardi refleja una práctica profesional estrechamente vinculada a la búsqueda del bien comunitario. En su primera etapa en Italia, trabajó como redactora en la revista Domus, entonces dirigida por Gio Ponti.
Ya desde sus inicios, la figura de Lina Bo Bardi es la de una mujer con una postura revolucionaria para su época (Sánchez Llorens, 2015). Cuando trabajó como directora del periódico femenino Grazia, en 1941, realizó un precioso proyecto de "cuna de emergencia" para gente sin recursos, el que ella calificó como su primer proyecto social. En la publicación explicaba cómo construir una cuna con una caja de frutas y algunos elementos más. A lo largo de su carrera profesional, encaró los problemas intelectuales del debate arquitectónico de la segunda mitad del siglo XX y teorizó en torno a las ideas que flotaban en el ámbito internacional, como aquellos temas propios de Brasil. En su discurso, la arquitectura, el arte, la cultura material y lo social, buscaban un terreno común capaz de dar respuesta a todas las necesidades de la época (Bardi, Rubino y Grinover, 2014: 29).

Teatro-Oficina en São Paulo es el estudio de caso expuesto. Se trata de un proyecto de restauración de un antiguo teatro, al igual que en SESC Pompeia y otros muchos proyectos desarrollados en Salvador de Bahía. Bardi decidió mantener el edificio existente y todo aqueIlo que ayudase a preservar la huella del lugar. Es paradójico cómo la obra de una arquitecta con esta actitud frente a la preservación resultó víctima de lo contrario. Sea como fuere, el proyecto Teatro-Oficina es la cristalización de múltiples experiencias vinculadas al mundo teatral. Diseñó escenografías, vestuario y tuvo contacto con muy distintos personajes involucrados en proyectos experimentales. El cineasta Glauber Rocha presentó a Lina Bo y a José Celso Martinez Corrêa, director de la compañía del Teatro Oficina y fundador, en 1967 (Bardi y Oliveira, 2002:184). El teatro había sufrido un incendio un año antes. El concepto espacial del nuevo teatro se planteó como una acera pública que lo cruzase desde su 

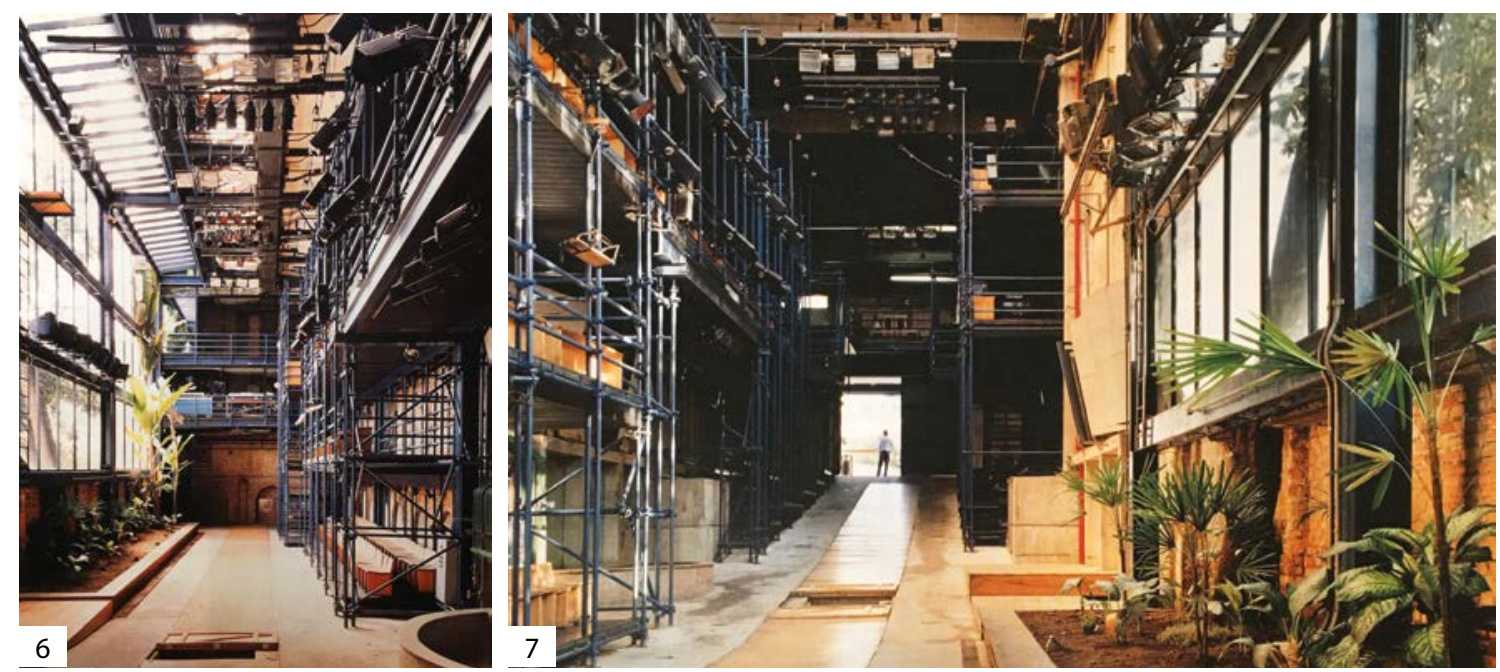

Figura 6 Teatro Oficina (1980-91), São Paulo. Arquitectos: Lina Bo Bardi y Edson Elito. Fuente: Fotografía de Nelson Kon. En: Bardi y Oliveira (2002: 188) / Figura 7 Teatro Oficina (1980-91), São Paulo. Arquitectos: Lina Bo Bardi y Edson Elito. Fuente: Fotografía de Nelson Kon. En: Bardi y Oliveira (2002: 187)

entrada, en la calle Jaceguai, hasta la calle Japurá, situada al fondo. Más tarde, con Lina al mando, y con la colaboración de Edson Elito, se decidió conservar solamente los muros de ladrillo visto del antiguo teatro, una caja de 9 metros de ancho y 50 de largo. El escenario se concibió como una rampa tipo calle-pasarela que abarca toda la longitud del teatro. En paralelo a los muros longitudinales, una estructura metálica azul desmontable genera unas galerías que ocupan la altura completa del edificio. Pueden ser ocupadas por el público, actores o técnicos, y funcionar como escenario, espacio para el público o camerinos (Figura 6 y 7 ).

Lina Bo Bardi proyectó un teatro en donde el espacio interior fue transformado en un lugar público que reproducía la vida urbana. En la propuesta no se planteaba el típico espacio de butacas. Es decir, estaba creando un espacio sin espectadores: actores, técnicos y público forman parte de la representación teatral junto a la propia arquitectura y los objetos que forman parte de la escenografía. Se evita a toda costa el modelo de teatro importado desde Europa en donde el público adquiere un rol pasivo como espectador, sin interacción con la función teatral. Se crea así un espacio que propicia un teatro dinámico, donde todas las partes participan de una misma experiencia. De hecho, el acceso desde la calle Jaceguai se realiza a través de un plano inclinado que forma parte de la escenografía (Figura 9). En el eje central del plano inclinado se incluye un suelo de madera, que funciona como caja de resonancia y consigue incorporarse como recurso sonoro. El público atraviesa este espacio y ocupa los laterales, como si de una calle se tratase. El suelo es desmontable, igual que los andamiajes que conforman las galerías. Lo preexistente y lo nuevo conviven, así como la arquitectura y la ciudad, los actores y el público. Todo forma parte de la propuesta teatral que se aleja de las historias míticas y los sueños imposibles para representar contenidos cercanos a la vida de la gente sencilla.

Según José Celso Martinez Corrèa, el teatro está concebido como un terreiro, donde antaño se oficiaba de manera clandestina el Camdomblé. El terreiro es también un espacio de tierra ubicado en las inmediaciones de la casa donde se desplazan muchas actividades de trabajo domésticas. Es el lugar donde se celebran fiestas y reuniones vecinales (Bardi y Oliveira, 2002).

Teatro-Oficina se conforma como un recinto lleno de referencias exteriores. Una calle-escenario con presencia del ambiente exterior: las superficies acristaladas que permiten la entrada del sol, la vegetación de gran porte plantada en suelo de tierra y la cubierta deslizante que da paso a la brisa y la lluvia (Figura 9). Incluso el andamiaje es un elemento propio del paisaje urbano. El teatro proyectado se funde con la trama urbana, generando un interfaz entre la ciudad y sus habitantes.

En un texto de Lina (1989) sobre sus obras en Salvador de Bahía, la arquitecta incluye una cita con una referencia a Le Corbusier que parece convertirse en la guía de su trabajo:

Teatro que sale a las plazas, a las calles, que invade la ciudad. Sillas y muebles que salen de las casas, y gente, hombres, mujeres, niños, todo un pueblo que inspiró, en 1963, a Le Corbusier, cuando visitaba Brasil, en una famosa carta al Ministro Gustavo Capanema: "Señor Ministro, no mande construir teatros con escenarios y butacas, deje las Plazas, las Calles, el Verde, libres. Mande únicamente construir "tablados" de madera, abiertos al Pueblo, y el Pueblo Brasileño los ocupará, "improvisando", con su elegancia natural y su inteligencia". (Bardi y Oliveira, 2002:184) 


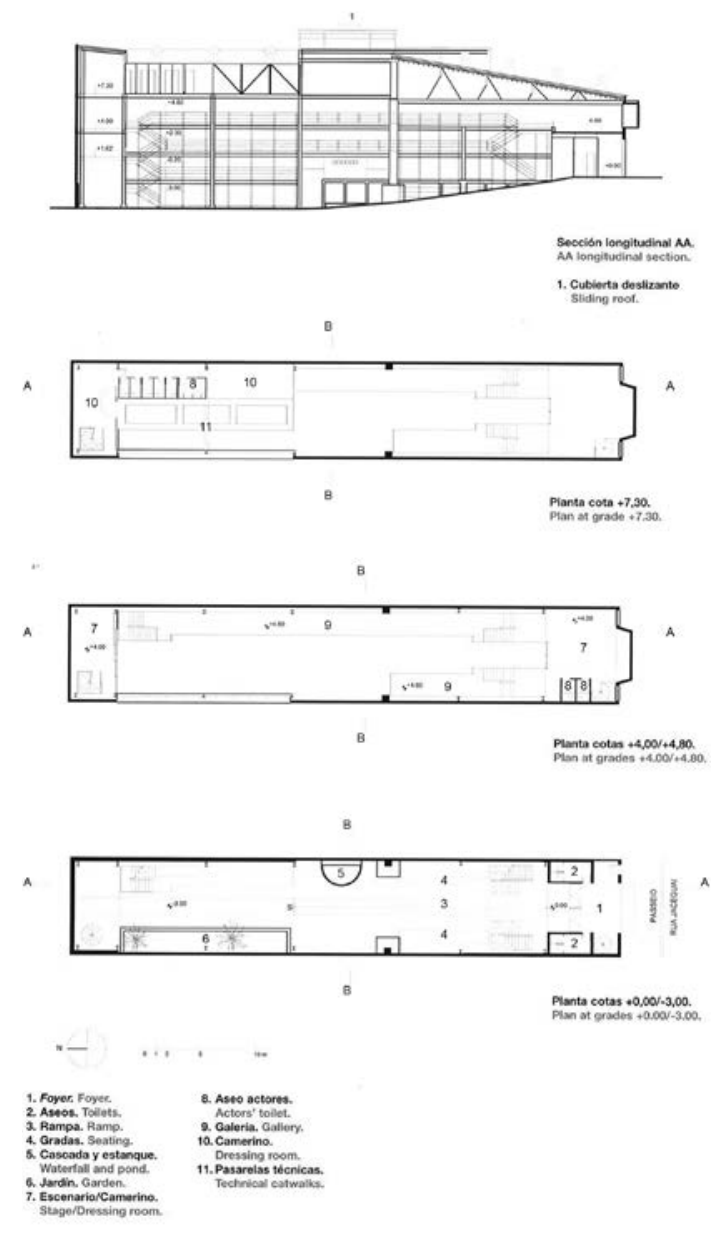

Figura 8 Plantas y sección Teatro Oficina (1980-91), São Paulo. Arquitectos: Lina Bo Bardi y Edson Elito. Fuente: Bard y Oliveira (2002: 186)

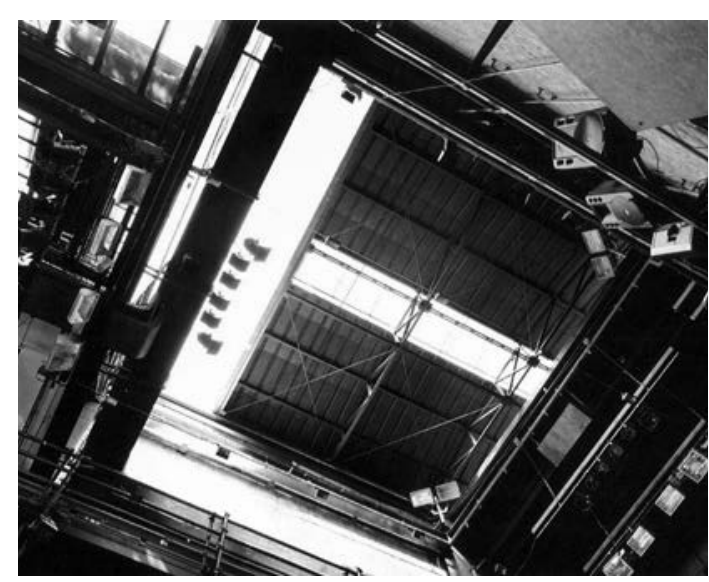

Figura 9 Teatro-Oficina (1980-91), São Paulo. Arquitectos: Lina Bo Bardi y Edson Elito. Fuente: Fotografía de Nelson Kon. En: Bardi y Oliveira (2002: 193).

\section{Isay Weinfeld}

El tercer y último estudio de caso atiende a un proyecto realizado por Isay Weinfeld, uno de los arquitectos contemporáneos más destacados del panorama actual brasileño. De origen polaco y en contacto con el mundo del cine junto a su colega Marcio Kogan, su arquitectura bebe de otras artes que forman parte de su visión global: «Para mí, la arquitectura es una forma de expresión, pero no es la única». A través de su arquitectura se puede observar cómo cada proyecto busca nuevas variables y formas de expresión: «trato de no aceptar trabajos que se relacionen con lo último que realicé... Quiero sorprenderme, arriesgar. Errar intentando algo nuevo antes que repetir una fórmula que funcione» (Weinfeld, Blanco y Fuentes, 2014: 16).

A diferencia de otros arquitectos históricos brasileños, su arquitectura no lleva implícita una crítica social, no hace alarde de pobreza y descarta el heroísmo estructural. Su arquitectura es contemporánea, elegante y sobria. Sus plantas de vivienda muestran una práctica arquitectónica en donde los espacios se activan mediante el mobiliario (en muchas ocasiones diseñado por él mismo) o prescinde de él en función del vacío a potenciar. Concentra y expande el programa en aras del habitar humano, haciendo uso del exterior, que se incluye en la vivencia interior. Hay un control total, desde lo más general hasta el ínfimo detalle, como si de un director de orquesta se tratase. $Y$ es que Weinfeld lo es: compositor y director. La sobriedad estética va acompañada de una definición exhaustiva que continúa la estela de arquitectos paulistas y otras figuras de la arquitectura brasileña del siglo XX, como Joaquim Guedes o Eduardo de Almeida, entre muchos otros.

El proyecto aquí seleccionado corresponde a una tienda de la marca de sandalias havaianas, que se ubica en una de las calles más caras de la ciudad de São Paulo, Rua Oscar Freire. La tienda se plantea como un espacio a doble altura excavado en el suelo (Figura 10). Cuando la tienda está abierta no existe ninguna fachada ni escaparate, sino que se convierte en una extensión de la vía urbana (Figura 11 y 12). La cubierta está formada por una retícula de celosías y lucernarios que inundan el espacio interior de luz natural, y en los laterales dos estrechas bandas de tierra incluyen vegetación que crece hasta techo. Todas las decisiones de proyecto buscan deshacer el espacio canónico de tienda y, en su lugar, se reproduce un escenario urbano ocupado por la marca. Con ello, el arquitecto busca generar un punto de venta que pueda transmitir el ambiente brasileño y no disociar este calzado de lo natural: «Nuestro mayor reto fue atrapar en la arquitectura el clima que la marca inspira: frescura, informalidad, comodidad, facilidad, bienestar, lo brasileño» (Weinfeld, 2009).

Los suelos se definen mediante solados de piedra y madera natural; escenarios habituales en donde se usan las havaianas. La marca exigía que la tienda fuese icónica dentro de la ciudad, para lo cual el arquitecto replicó el punto de venta más primitivo del famoso calzado: los mercados urbanos. En tal dirección, incluye otros elementos independientes que implementan la experiencia de compra: un cubo a modo de expositor recoge la historia de la marca, un contenedor expone modelos importados y un cilindro transparente presenta la nueva colección. De esta manera, se consigue activar el suelo de planta e incorporar la filosofía de marca al espacio dedicado a la tienda, estableciendo un nuevo tipo de relación con el cliente e incluyendo una nueva experiencia espacial asociada a la compra. Mediante la diferencia de cotas y la supresión de la fachada se logra generar un recinto espacial en continuo contacto con el exterior. No existe un límite claro que defina la acción de "entrar"; se convierte, en cambio, en un fenómeno gradual que descubre un espacio urbano más conectado a la ciudad. 


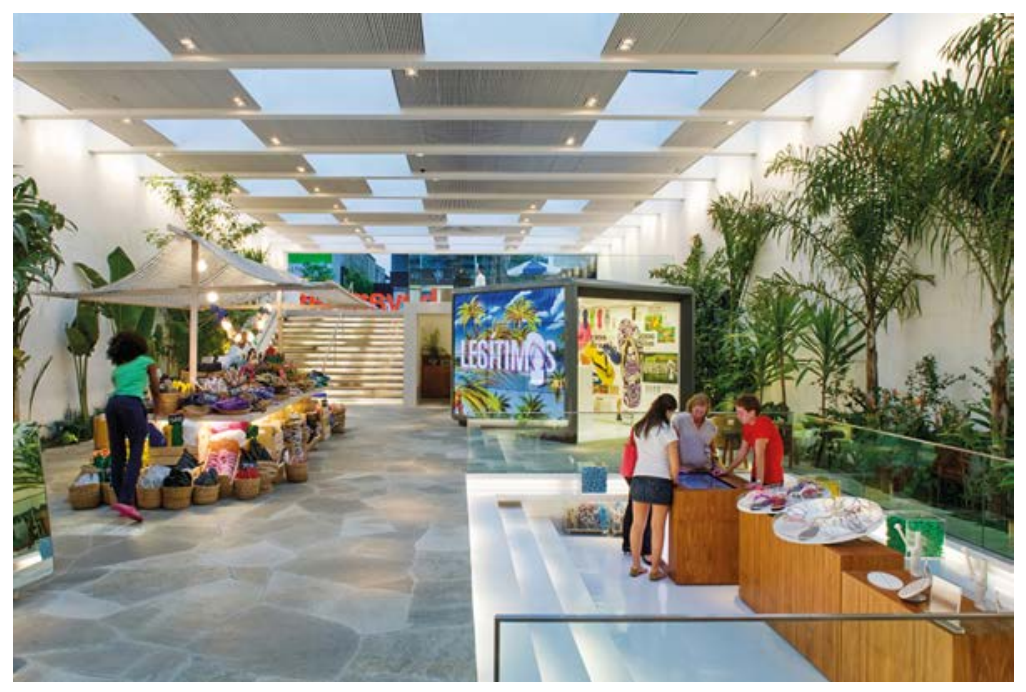

Figura 10 Tienda Havaianas (2009), São Paulo. Arquitecto: Isay Weinfeld. Fuente: Fotografía de Nelson Kon (http:// www. plataformaarquitectura.cl)

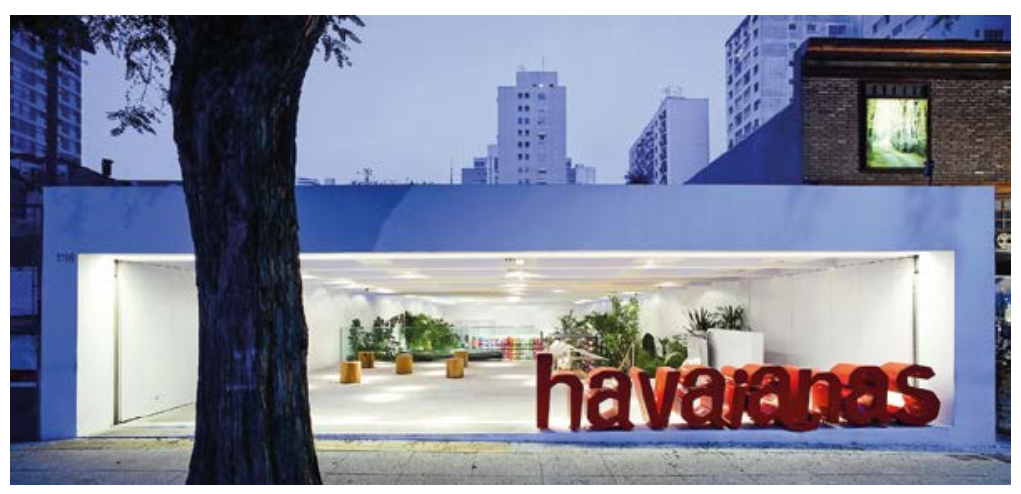

Figura 11 Tienda Havaianas (2009), São Paulo. Arquitecto: Isay Weinfeld. Fuente: Fotografía de Nelson Kon (https://arcoweb.com.br)

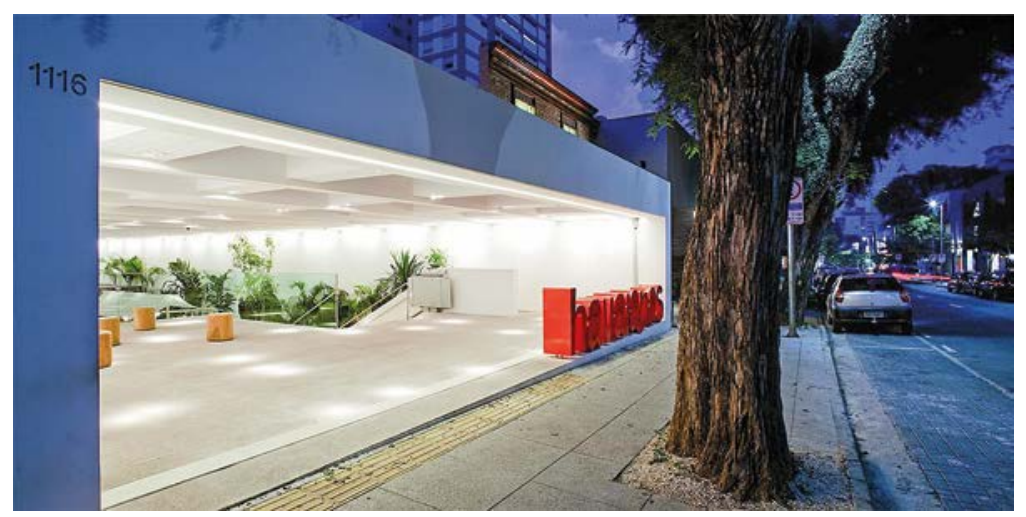

Figura 12 Tienda Havaianas (2009), São Paulo. Arquitecto: Isay Weinfeld. Fuente: Fotografía de Nelson Kon (https://arcoweb.com.br) 


\section{CONCLUSIONES}

Los tres casos de estudio presentados en este artículo abordan, desde distintas perspectivas, maneras de transformar espacios interiores con usos concretos. Asimismo, se pone en valor aquí el espacio público desde intervenciones que se realizan dentro de la arquitectura. La dimensión urbana no se debe plantear como algo ajeno a la arquitectura que conforma la ciudad, y para ello las distintas tentativas expuestas muestran trabajos en donde lo público y lo privado dejan de marchar en paralelo para establecer una relación de simbiosis. Esto es, ambos mundos salen beneficiados gracias al vínculo establecido.

Desde esa perspectiva, es imprescindible redefinir el concepto de uso, ofreciendo nuevos escenarios que animan vivencias dinámicas mucho más centradas en la vivencia en común. Mendes da Rocha, mediante la definición de los límites del dormitorio, modela un sistema de relaciones que se distancia del individualismo, propiciando lo comunitario. Impregna, de esa forma, el recinto espacial doméstico de valores que tienen que ver con la ética social, con aquello que hace que una comunidad se desarrolle mediante un correcto funcionamiento. Una dimensión intangible de lo urbano y lo colectivo se reincorpora ${ }^{10}$ al espacio de vivienda.

Ocurre lo mismo en los otros dos casos analizados. Tanto la experiencia de compra diseñada por Weinfeld, como el modelo de teatro de Bardi van asociados a la propuesta espacial. Lina Bo desarrolla un espacio en donde, además de la conexión física con el ambiente exterior, se crea una calle domesticada y versátil capaz de adaptarse a celebraciones comunitarias que utilizan el teatro para poner en valor y expresar vivencias e inquietudes propias. El teatro deja de plantearse como un edificio ensimismado que ignora la ciudad y se funde con ella con el fin de crear una experiencia colectiva y cercana.

Weinfeld también modifica la experiencia de compra asociada a la tienda. Recupera el concepto de mercado y se desvincula del espacio privatizado, cuya definición material puede llevar implícito un mensaje de exclusión. Las havaianas representan el calzado económico y asequible del pueblo. No existen barreras que impidan el acceso, o al menos la supresión de éstas consiguen plantear una alternativa más amable que la fachada canónica, en donde la acción de acceder es mucho más evidente y exclusiva. En el proceso evolutivo de la ciudad, la conquista del espacio urbano como una propiedad colectiva es uno de los caminos a recorrer por nuestra sociedad. Reproduciendo un paisaje urbano en un espacio interior se deshacen los límites más abstractos que asocian códigos y símbolos a ciertos estatus que excluyen y marginan. Con los tres proyectos destacados, se están dando a conocer tres iniciativas que proponen un contrapunto y se establecen como resistencias que bien podrían ser el germen de tendencias futuras.
Es importante señalar que todos los casos de estudio intentan recuperar modelos del pasado, en donde el uso del espacio público y las relaciones comunitarias tenían un mayor peso dentro de los colectivos humanos. La cultura occidental, la privatización y la explotación económica de ciertos sectores llevan asociada una formalización espacial basada en el refuerzo del límite como elemento que disocia los espacios interiores con respecto a la ciudad. Incluso el modelo de familia occidental también propicia el individualismo y esto afecta a las relaciones entre personas y con la sociedad. A través de la manipulación espacial y sus límites es posible crear arquitectura y ciudad basadas en una relación de simbiosis que propicia la vida comunitaria, evitando disociar dos entidades espaciales que encuentran en su asociación, una mejor alternativa para el desarrollo de la vida comunitaria. 
ANDREOLI, Elisabetta y FORTY Adrian. Brazil's modern architecture. London: Phaidon, 2004.

BARDI, Lina Bo. "Pequena comunicação sobre restauro na arquitetura na Bahia". Archivo Instituto Lina Bo e P.M. Bardi, Evora, septiembre 1989. En: BARDI, Lina Bo y OLIVEIRA, Olivia de. Lina Bo Bardi: obra construída = built work. Barcelona: Gustavo Gili, 2002.

BARDI, Lina Bo y OLIVEIRA, Olivia de. Lina Bo Bardi: obra construída = built work. Barcelona: Gustavo Gili, 2002.

BARDI, Lina Bo, RUBINO, Silvana y GRINOVER, Marina. Lina bo Bardi por escrito: textos escogidos 1943-1991. México: Alias, 2014. pp.28-35

DECKKER, Zilah Quezado. Brazil built: the architecture of the modern movement in Brazil. London: Spon Press, 2001.

FUTAGAWA, Yoshio. Circumstances surrounding Japanese houses today. GA Houses, 2007, n¹00, pp. 177-187.

GARCÍA DEL MONTE, José María. Paulo Mendes Da Rocha: conciencia arquitectónica del pretensado. Buenos Aires: Nobuko, 2011.

GUTIÉRREZ, Ramón. Arquitectura y urbanismo en lberoamérica. Madrid: Cátedra, 1983.

MASAO, João. The Modern Brasil House. En: ANDREOLI, Elisabetta y FORTY Adrian. Brazil's modern architecture. London: Phaidon, 2004, pp.142-170

MENDES da Rocha, Paulo. Paulo Mendes da Rocha, 19582013. Arquitectura Viva, 2013, nº 161.

MONTEZUMA, Roberto. Arquitectura brasil 500 anos. Una investigacáo reciproca. Recife: Universidade Federal de Pernambuco, 2002.

PIÑÓN, Helio. Paulo Mendes da Rocha. Barcelona: UPC, 2003.

SÁNCHEZ LLORENS, Mara. Lina Bo Bardi. Objetos y acciones colectivas. Buenos Aires: Diseño Editorial, 2015.

WATSUJI, Tetsuro. Antropología del paisaje: climas, culturas y religiones. Salamanca: Ediciones Sígueme, 2006.

WEINFELD, Isay. Tienda Havaianas [en línea], septiembre 2009. [Consultado 6 febrero 2017]. Disponible en: http:// www.plataformaarquitectura.cl/cl/02-26007/tiendahavaianas-isay-weinfeld

WEINFELD, Isay, BLANCO, Ricardo y FUENTES, Oscar. Isay Weinfeld. Buenos Aires: [1:100], 2014. 